\title{
Coordinates Determination of Submerged Sensors Using Cayley-Menger Determinant
}

\author{
Anisur Rahman ${ }^{1}$, Vallipuram Muthukkumarasamy ${ }^{2}$, Elankayer Sithirasenan ${ }^{3}$ \\ School of Information and Communication Technology \\ Griffith University \\ Gold Coast, Australia \\ $\left\{{ }^{1}\right.$ anis.rahman, ${ }^{2}$ v.muthu, ${ }^{3}$ e.sithirasenan $\} @$ griffith.edu.au
}

\begin{abstract}
This paper investigates the problem of localizing submerged sensors and provides a new mechanism to determine the coordinates of those sensors using only one beacon node. In underwater wireless sensor networks (UWSN), precise coordinate of the sensors that actuate or collect data is vital, as data without the knowledge of its actual origin has limited value. Mostly, multilateration technique is used to determine the location of the sensors with respect to three or more known beacon nodes where distance between them is measured considering the roundtrip time of acoustic signal. However, this method of measuring distances gives erroneous results due to a number of factors, including relative angular stand of the nodes. In this study, a new method of determining the underwater distances between beacon and sensor nodes has been presented using combined radio and acoustic signals, which has better immunity from multipath fading. Moreover, Cayley-Menger determinant is used to determine the coordinates of the nodes where none of nodes have a priori knowledge about its location. Simulation results validate the proposed mathematical models by computing coordinates of sensor nodes with negligible errors.
\end{abstract}

Index Terms-localization, Cayley-Menger determinant, underwater wireless sensor networks, linearization.

\section{INTRODUCTION}

While wireless communication has become more prominent in terrestrial applications, challenges still exist in submerged sensor fields. In the recent years, there have been growing interests to explore and address the needs of multitude of underwater applications, including: oceanographic data collection, marine ecology, offshore exploration, underwater surveillance etc. Marine life is a vast resource providing food, medicine, and raw materials, in addition to helping to support the ecosystem, recreation and tourism around the world. It is, therefore, crucial to collect accurate environmental data using underwater sensors, and to develop appropriate mechanisms for actuation tasks. Depending on the type of deployment, the wireless sensor and actuator network may consist of surface monitoring stations, autonomous underwater vehicles and various environmental sensors. In most of these applications (indoor, outdoor, underground or underwater) the actual location of sensors and actuators needs to be determined accurately for proper control and operation, and meaningful interpretation of the sensed data [1].

Localization has widely been explored in terrestrial wireless sensor network (WSN) and various mechanisms have been proposed. Generally these methods can be classified into two categories: range-based and range-free schemes. The former apply inter node distances to multilateration or triangulation whereas the latter rely on profiling. Range-based scheme can provide more accurate position estimation with additional hardware for distance measurement, which most sensors now have that provision. In this paper, we focus on accurate localization in underwater, which is needed in a range of applications, such as estuary monitoring and pollutant tracking [2]. Moreover, in UWSNs, acoustic channels are naturally employed, and range measurement using acoustic signals are more accurate than using radio $[2,3]$.

In [4], 3D Euclidean distance estimation method requires the need of a certain number of neighboring nodes to measure inter-node distances and where error is propagated through the system due to its recursive nature. In [5], the authors propose a localization scheme based on buoys moored to the waterbed and mobile nodes that need to communicate directly with these buoys to get their location. This method does not support dynamic environment because buoys need to be deployed in advance in known locations. In [6], four different positions are used to obtain the beacon nodes positions of a 3D local positioning system (LPS).

In [7], Duff and Muller proposed a method to solve the multilateration equations by means of nonlinear least square optimization when positions are not known. The algorithm is based on degree-of-freedom analysis - which says enough measurements from different positions will provide enough equations to solve the problem. In [8], same technique is used incorporating extended Kalman filter. However, the degree-of-freedom analysis does not guarantee a unique solution in a system on nonlinear equations, such as trilateration, when the only data available is the distance measured between the nodes [9]. In [10], Guevara et al. introduced a new closed-form solution where no position information of nodes is required to determine the positions of multiple static beacon nodes, the only information they 
used is the distance measurement between static beacons and mobile node.

Having analyzed the various studies discussed above, in this paper we propose a closed-form solution to determine the coordinates of the submerged sensors having only one beacon node at the surface. The precise conditions for obtaining initial subsets of nodes were justified using rigidity theory in [9]. Section II describes the problem domain, environmental constraints and Section III describes the proposed distance measurement technique. Section IV explains the proposed theoretical mechanism to determine the sensors coordinates. Analysis is given in Section V. Simulation results and discussions are reported in Section VI and finally conclusions in Section VII.

\section{PROPOSED CONFIGURATION}

\section{A. Problem Domain}

To determine the coordinates of the submerged sensors, the proposed method assumes at least three sensors and a floating beacon. It is also assumed that the distance measurement between the beacon and sensors are possible, as described in Section III. In the marine environment, a boat or a buoy can be used as a beacon and sensors could be deployed in the water. While measuring the multiple distances between the beacon and sensors, those locations of the beacon are assumed to be in a plane, which is approximately parallel to the plan created by the three sensors (as shown in Fig. 1).

For simplicity, at present we assume that the submerged sensors are stationary for a short period of time, during which the measurements of the distances from six different locations of the beacon are taken. As the general properties of a transducer, beacon has the capability of generating radio and acoustic signal, whereas sensors might have the restricted capability of receiving the radio and acoustic signal for timing purpose as well as it is enabled with acoustic transmission. A solvable configuration of one beacon with three submerged sensors is denoted in Fig. 1. Our proposed mechanism exploits the advantage of both radio and acoustic signal propagation in sea water in $1.8-323 \mathrm{~m}$ depth [11]. Since most of the marine explorations take place in shallow water, our proposed model has wide ranging practical applications.

\section{B. Environmental Constrains}

Normally, underwater environment is more adverse than terrestrial environment; despite those limitations, it poses some merits that could be exploited in determining coordinates. Water body is relatively more homogeneous because the usual obstacles present in water are smaller in size than that of in terrestrial environment. The region of interest on the ground is more likely occupied with buildings and trees which are the major factors for multipath propagation.

Regarding signal propagation in water, acoustic signal propagates much further compare to radio signal; however,

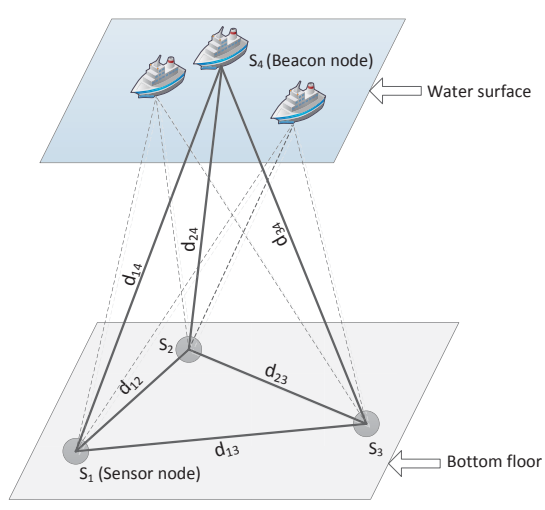

Fig. 1. Subset composed of one beacon and three submerged sensors

the speed of the acoustic signal is much slower than that of radio signal. TABLE I shows some limitations and typical measurements for radio and acoustic signals.

The main environmental variable that we assume in our method to determine distances is the speed of acoustic signals in water. It depends on the temperature, salinity and permeability. How the speed of acoustic will vary because of aforesaid factors is not considered in this study, but our mathematical model assumes it as a variable $v_{A}$.

TABLE I. Properties of RAdio and Acoustic Signals

\begin{tabular}{|c|c|c|c|c|}
\cline { 2 - 5 } \multicolumn{1}{c|}{} & \multicolumn{2}{c|}{ Radio Signal } & \multicolumn{2}{c|}{ Acoustic Signal } \\
\cline { 2 - 5 } \multicolumn{1}{c|}{} & Vacuum & Water & Vacuum & Water \\
\hline Velocity & $3 \times 10^{8} \mathrm{~m} / \mathrm{s}$ & $\approx 2.25 \times 10^{6} \mathrm{~m} / \mathrm{s}$ & $\approx 1500 \mathrm{~m} / \mathrm{s}$ & $\mathrm{V}_{\mathrm{A}}$ \\
\hline Range & - & $1.8-323 \mathrm{~m}$ & - & $1-100 \mathrm{~km}$ \\
\hline
\end{tabular}

\section{Distance DETERMINATION}

Despite the limitation of both radio and acoustic signal propagation in water in different aspect, we will exploit each of their merits in our proposed method to increase the accuracy of the distance measurements. Differential speed between radio and acoustic signals will be used to calculate the distance, while acoustic signal will be used for communication purposes. This method will require a short communication in between the beacon and sensor nodes.

Even though the speed of radio signal is slightly less than that of in the vacuum, considering the problem domain, the speed variation will not have significant impact on the proposed localization method. Moreover, the speed of acoustic signal, which varies due to different environmental factors, is the main variable that we need to use for coordinate determination.

1) Assumptions:

- The beacon can generate radio and acoustic signals simultaneously.

- The environmental factors that affect the acoustic signal will be considered while measuring internode distances.

- Sensor nodes are stationary during the short measurement period.

- Each sensor node will have a unique ID. 


\section{2) Steps:}

i. Simultaneous generation of radio and acoustic signals by the beacon $S_{j}, j=4,5 \ldots$ at $t_{0}$

ii. For any submerged sensors $S_{i}, i=1,2,3$

a. Sensors receive the radio signal at $t_{R(\text { rec })}=t_{0}+\varepsilon$

b. Sensor receives the acoustic signal at $t_{A(\text { rec })}$; here $t_{A(\text { rec })} \gg t_{R(\text { rec })}$

iii. Time taken for the acoustic signal to travel from beacon to sensors is:

$$
\begin{aligned}
& T_{i j(\text { Travel }), i=1,2,3 ; j=4,5,6 \ldots . . .}=t_{A(\text { rec })}-t_{A(\text { tra })} \\
& =t_{A(\text { rec })}-t_{R(\text { tra })} \because t_{A(\text { tra })}=t_{R(\text { tra })} \\
& \therefore T_{i j \text { (Trave) })} \approx t_{A(\text { rec })}-t_{R(\text { (rec })} \because t_{R(\text { rec })}=t_{0}+\varepsilon \approx t_{R(\text { ra } a)}
\end{aligned}
$$

iv. Sensor nodes send back the time $T_{i j(\text { Travel })}$ with individual sensor's ID to the beacon using acoustic signal.

v. Beacon node computes the distance between the beacon and sensors: $d_{i j}=v_{A} \times T_{i j(\text { travel) }}$ here, $v_{A}$ is average acoustic signal speed.

\section{COORDINATES COMPUTATION}

\section{A. Coordinates of the Sensors}

The objective of localization algorithms is to obtain the exact position or coordinates of all the sensor nodes by measuring distances between beacon and them. Only measurements available here to compute is the distance and typically it is considered as optimization problem where objective functions to be minimized have residuals of the distance equations. The variables of any localization problem are the $3 \mathrm{D}$ coordinates of the nodes. In principle more number of distance equations are needed than number of variables to solve this kind of problem. However, this approach known as degree of freedom analysis may not guarantee the unique solution in a nonlinear system.

Trilateration or multilateration techniques that are nonlinear system usually used to determine the location or coordinates of the sensors in partial or full. According to Guevara et al. [10] the convergence of optimization algorithms and Bayesian methods depend heavily on initial conditions used and they circumvent the convergence problem by linearizing the trilateration equations.

Fig. 2 shows the initial subset composed of the beacon node $S_{j}, j=4,5 \ldots .9$ and three sensor nodes $S_{i}, i=1,2,3$. Without loss of generality, a coordinate system can be defined using one of the sensor nodes $S_{i}, i=1,2,3$. as the origin $(0,0,0)$ of the coordinate system. Now the trilateration equations can be written as a function of two groups of distance measurements. The distance between beacon and sensors $d_{14}, d_{24}, d_{34} \ldots$ which are measured data, and inter- sensor distances $d_{12}, d_{13}, d_{23}$ and the volume of tetrahedron $V_{t}$ (formed by the beacon and sensors), are unknown.

By expanding and grouping known-unknown variables of (1), we obtain;

$$
\begin{aligned}
& d_{34}^{2}\left(d_{12}^{2}-d_{23}^{2}-d_{13}^{2}\right)+d_{14}^{2}\left(\frac{d_{23}^{4}}{d_{12}^{2}}-d_{23}^{2}-\frac{d_{11}^{2} d_{23}^{2}}{d_{12}^{2}}\right)+d_{24}^{2}\left(\frac{d_{13}^{4}}{d_{12}^{2}}-\frac{d_{11}^{2} d_{23}^{2}}{d_{12}^{2}}-d_{13}^{2}\right) \\
& -\left(d_{14}^{2} d_{24}^{2}+d_{14}^{2} d_{34}^{2}-d_{24}^{2} d_{34}^{2}-d_{14}^{4}\right) \frac{d_{23}^{2}}{d_{12}^{2}}-\left(d_{34}^{2} d_{24}^{2}-d_{14}^{2} d_{34}^{2}+d_{14}^{2} d_{24}^{2}-d_{24}^{4}\right) \\
& \frac{d_{13}^{2}}{d_{12}^{2}}+\left(144 \frac{V_{2}^{2}}{d_{12}^{2}}+d_{13}^{2} d_{23}^{2}\right)=\left(d_{24}^{2} d_{34}^{2}-d_{34}^{4}+d_{14}^{2} d_{34}^{2}-d_{14}^{2} d_{24}^{2}\right)
\end{aligned}
$$

Here, $\quad\left(d_{12}^{2}-d_{23}^{2}+d_{13}^{2}\right), \quad\left(\frac{d_{23}^{2}}{d_{12}^{2}}-d_{23}^{2}-\frac{d_{13}^{2} d_{23}^{2}}{d_{12}^{2}}\right), \quad\left(\frac{d_{13}^{4}}{d_{12}^{2}}-\frac{d_{13}^{2} d^{2} 2}{d_{12}^{2}}-d_{13}^{2}\right)$ $\frac{d_{23}^{2}}{d_{12}^{2}}, \frac{d_{13}^{2}}{d_{12}^{2}}$ and $\left(144 \frac{V_{t}^{2}}{d_{12}^{2}}+d_{13}^{2} d_{23}^{2}\right)$ are unknown terms.

The above expansion can be rewritten as follows:

$$
\begin{aligned}
& d_{14}^{2} X_{1}+d_{24}^{2} X_{2}+d_{34}^{2} X_{3}-\left(d_{14}^{2}-d_{34}^{2}\right)\left(d_{24}^{2}-d_{14}^{2}\right) X_{4} \\
& -\left(d_{24}^{2}-d_{14}^{2}\right)\left(d_{34}^{2}-d_{24}^{2}\right) X_{5}+X_{6}=\left(d_{24}^{2}-d_{34}^{2}\right)\left(d_{34}^{2}-d_{14}^{2}\right)
\end{aligned}
$$

Based on the local positioning system configuration of Fig. 2, we need to write equations that will include all known and unknown distances. For that matter, we express the volume of tetrahedron $V_{t}$ using Cayley-Menger determinant as following:

$$
288 V_{t}^{2}=\left|\begin{array}{ccccc}
0 & 1 & 1 & 1 & 1 \\
1 & 0 & d_{12}^{2} & d_{13}^{2} & d_{14}^{2} \\
1 & d_{12}^{2} & 0 & d_{23}^{2} & d_{24}^{2} \\
1 & d_{13}^{2} & d_{23}^{2} & 0 & d_{34}^{2} \\
1 & d_{14}^{2} & d_{24}^{2} & d_{34}^{2} & 0
\end{array}\right|
$$

The Equation (2) in fact resembles the linear form of $a_{1} x_{1}+a_{2} x_{2}+\cdots+a_{n} x_{n}=b_{1}$. As we have six unknown in (1), we need at least six measurements, which could be done following the same procedure described in Section III steering the beacon node $S_{j}, j=4,5 \ldots .9$ to six different locations and measuring the distances in the vicinity of $S_{4}$.

Finally, we get m-linear equations of the form;

$$
\begin{gathered}
a_{11} x_{1}+a_{12} x_{2}+\cdots+a_{1 n} x_{n}=b_{1}, \\
a_{21} x_{1}+a_{22} x_{2}+\cdots+a_{2 n} x_{n}=b_{2}, \\
\vdots \\
a_{m 1} x_{1}+a_{m 2} x_{2}+\cdots+a_{m n} x_{n}=b_{m},
\end{gathered}
$$

If we omit reference to the variables, then system of equations in (3) can be represented by the array of all coefficients known as the augmented matrix of the system, where the first row of the array represents the first linear equation and so on. That could then be expressed in $A X=b$ linear form.

Then, the system of equations can be written as:

$$
A=\left[\begin{array}{cccccc}
d_{14}^{2} & d_{24}^{2} & d_{34}^{2} & -\left(d_{14}^{2}-d_{34}^{2}\right)\left(d_{24}^{2}-d_{14}^{2}\right) & -\left(d_{24}^{2}-d_{14}^{2}\right)\left(d_{34}^{2}-d_{24}^{2}\right) & 1 \\
d_{15}^{2} & d_{25}^{2} & d_{35}^{2} & -\left(d_{15}^{2}-d_{35}^{2}\right)\left(d_{25}^{2}-d_{15}^{2}\right) & -\left(d_{25}^{2}-d_{15}^{2}\right)\left(d_{35}^{2}-d_{25}^{2}\right) & 1 \\
\vdots & \vdots & \vdots & \vdots & \vdots & \vdots \\
d_{19}^{2} & d_{29}^{2} & d_{39}^{2} & -\left(d_{19}^{2}-d_{39}^{2}\right)\left(d_{29}^{2}-d_{19}^{2}\right) & -\left(d_{29}^{2}-d_{19}^{2}\right)\left(d_{39}^{2}-d_{29}^{2}\right) & 1
\end{array}\right]
$$




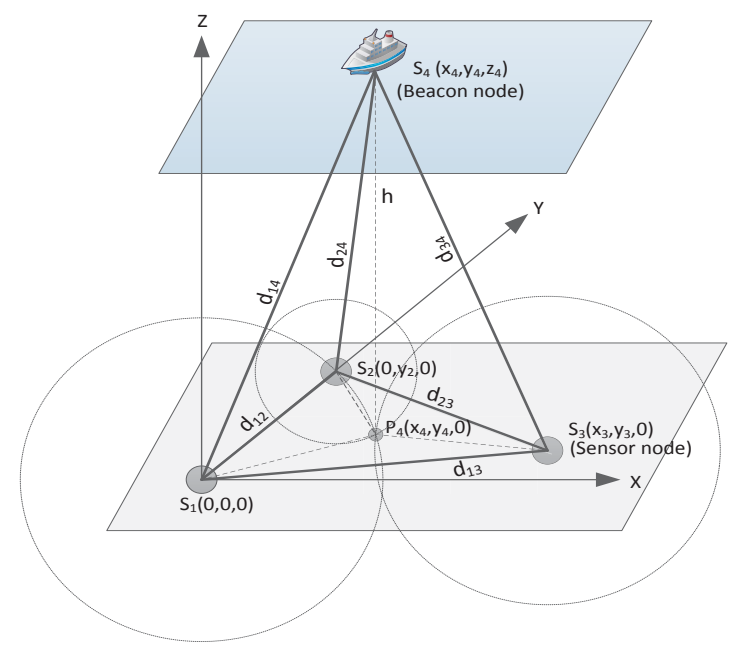

Fig. 2. Coordinates determinations

$$
X=\left[\begin{array}{c}
\left(\frac{d_{23}^{4}}{d_{12}^{2}}-d_{23}^{2}-\frac{d_{13}^{2} d_{23}^{2}}{d_{12}^{2}}\right) \\
\left(\frac{d_{13}^{4}}{d_{12}^{2}}-\frac{d_{13}^{2} d_{23}^{2}}{d_{12}^{2}}-d_{13}^{2}\right) \\
\left(d_{12}^{2}-d_{23}^{2}-d_{13}^{2}\right) \\
\frac{d_{23}^{2}}{d_{12}^{2}} \\
\frac{d_{13}^{2}}{d_{12}^{2}} \\
\left(144 \frac{V_{t}^{2}}{d_{12}^{2}}+d_{13}^{2} d_{23}^{2}\right)
\end{array}\right] \quad b=\left[\begin{array}{c}
\left(d_{24}^{2}-d_{34}^{2}\right)\left(d_{34}^{2}-d_{14}^{2}\right) \\
\left(d_{25}^{2}-d_{35}^{2}\right)\left(d_{35}^{2}-d_{15}^{2}\right) \\
\vdots \\
\left(d_{29}^{2}-d_{39}^{2}\right)\left(d_{29}^{2}-d_{19}^{2}\right)
\end{array}\right]
$$

From the above representation, after finding $X_{1}, X_{2}, X_{3}$, $X_{4}, X_{5}$ and $X_{6}$ we calculate $d_{12}, d_{13}$ and $d_{23}$ as follows:

$d_{12}^{2}=\frac{X_{3}}{\left(1-X_{4}-X_{5}\right)}, d_{13}^{2}=\frac{X_{3} X_{5}}{\left(1-X_{4}-X_{5}\right)}, d_{23}^{2}=\frac{X_{3} X_{4}}{\left(1-X_{4}-X_{5}\right)}$

If we let the coordinates of the submerged sensors $S_{1}, S_{2}$ and $S_{3}$ are $(0,0,0),\left(0, y_{2}, 0\right)$ and $\left(x_{3}, y_{3}, 0\right)$ respectively, then the inter-sensor distances could be written with respect to coordinates of the sensors as follows:

$$
d_{12}^{2}=y_{2}^{2}, d_{13}^{2}=x_{3}^{2}+y_{3}^{2}, d_{23}^{2}=x_{3}^{2}+\left(y_{3}-y_{2}\right)^{2}
$$

From the above values the unknown variables can be computed as follows:

$$
y_{2}=d_{12}, y_{3}=\frac{d_{12}^{2}+d_{13}^{2}-d_{23}^{2}}{2 d_{12}}, x_{3}=\sqrt{\left(d_{13}^{2}-\left(\frac{d_{12}^{2}+d_{13}^{2}-d_{23}^{2}}{2 d_{12}}\right)^{2}\right)}
$$

where $d_{12}, d_{13}$ and $d_{23}$ are known computed distances. TABLE II summarizes the coordinates of the sensors for this system.

\section{B. Coordinates of the Sensors with Respect to the Beacon}

$\mathrm{Up}$ to now we have been able to determine the coordinates of the sensor nodes with respect to $S_{1}$. In order to find the coordinate with respect to the beacon node we follow the following steps.
TABLE II. COORDINATES OF THE SENSORS WITH KNOWN MEASUREMENTS

\begin{tabular}{|c|c|}
\hline Sensors & Coordinates \\
\hline$S_{1}$ & $(0,0,0)$ \\
\hline$S_{2}$ & $\left(0, d_{12}, 0\right)$ \\
\hline$S_{3}$ & $\left(\sqrt{\left(d_{13}^{2}-\left(\frac{d_{12}^{2}+d_{13}^{2}-d_{23}^{2}}{2 d_{12}}\right)^{2}\right)}, \frac{d_{12}^{2}+d_{13}^{2}-d_{23}^{2}}{2 d_{12}}, 0\right)$ \\
\hline
\end{tabular}

We assume that with the use of appropriate sensors, the depth $h$ can be measured [12]. After measuring the vertical distance $h$ in between the beacon node $S_{4}\left(x_{4}, y_{4}, z_{4}\right)$ and the $X Y$ plane, we can assume the projected coordinate of the beacon node $S_{4}\left(x_{4}, y_{4}, z_{4}\right)$ on the plan $X Y$ is $P_{4}\left(x_{4}, y_{4}, 0\right)$. To find $x_{4}$ and $y_{4}$, we can apply trilateration in the following manner assuming the distances between $S_{1}, S_{2}, S_{3}$ and $P_{4}$ are $D_{14}, D_{24}$ and $D_{34}$ respectively.

$$
\begin{gathered}
D_{14}^{2}=x_{4}^{2}+y_{4}^{2} \\
D_{24}^{2}=x_{4}^{2}+\left(y_{4}-y_{2}\right)^{2} \\
D_{34}^{2}=\left(x_{4}-x_{3}\right)^{2}+\left(y_{4}-y_{3}\right)^{2}
\end{gathered}
$$

From equation (4), (5) and (6) we obtain the projected beacon's coordinates $P_{4}\left(x_{4}, y_{4}, 0\right)$.

$x_{4}=\sqrt{\frac{1}{2 D}\left(2 d_{12} D_{14}^{2}-D_{14}^{2}+D_{24}^{2}+d_{12}^{2}\right)}, y_{4}=\frac{1}{2 d_{12}}\left(D_{14}^{2}-D_{24}^{2}+d_{12}^{2}\right)$

As $d_{14}, d_{24}$ and $d_{34}$ are the hypotenuse of the $\Delta S_{1} P_{4} S_{4}$, $\Delta S_{2} P_{4} S_{4}$ and $\Delta S_{3} P_{4} S_{4}$ respectively, so it is possible to obtain $D_{14}, D_{24}$ and $D_{34}$ using Pythagorean Theorem. So the coordinate of the beacon node $S_{4}\left(x_{4}, y_{4}, z_{4}\right)$ would be $\left(x_{4}, y_{4}, h\right)$ where all the elements are known.

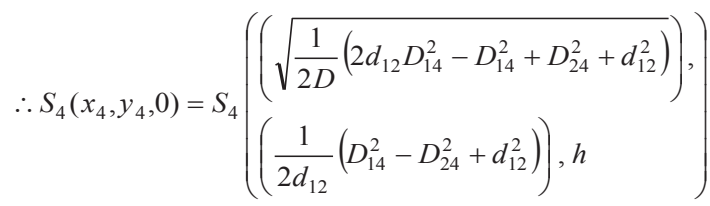

If the origin of the Cartesian system is transferred on to the coordinate of the beacon node, then it is possible to find the coordinates of other sensors with respect to the beacon node $S_{4}$. A linear transformation would give the results as in TABLE III.

TABLE III. COORDINATES OF THE SENSORS WITH RESPECT TO BEACON

\begin{tabular}{|c|c|c|c|}
\hline Sensors & Coordinates & Sensors & Coordinates \\
\hline$S_{4}$ & $(0,0,0)$ & $S_{2}$ & $\left(-x_{4}, y_{2}-y_{4},-z_{4}\right)$ \\
\hline$S_{1}$ & $\left(-x_{4},-y_{4},-z_{4}\right)$ & $S_{3}$ & $\left(-x_{4}, y_{2}-y_{4},-z_{4}\right)$ \\
\hline
\end{tabular}

\section{ANALYSIS}

The proposed method is for a specific configuration and scenario, where a single beacon node is necessary to determine the coordinates of the sensors. Usually numerous beacons and sensors are deployed to localize and most methods rely on distance measurements, thus the precision 
of distance measurement is one of the prime factors for accurate coordinate determination.

In our proposed approach the number of beacons required is just one that floats (to six locations in proximity) on the surface of the water and minimum of three sensors - a recognized number in monitoring for analyzing environment with sensors. Besides, our method is capable of determining $3 \mathrm{D}$ coordinates with respect to the beacon node. It gives a better comprehension regarding the locations of the sensors because coordinates of the beacon node could be known with the help of GPS.

\section{A. Complexity of Distance Measurement}

Being aware of the limitations of radio and acoustic signals in water, each of its merit has been used in our proposed method to determine the distances in the problem domain. The method is relatively simple but precise enough when both the beacon and sensors are capable of transmitting/receiving radio and acoustic signals. To be precise, the beacon should be capable of acoustic (transmit $\left(T_{x}\right) \&$ receive $\left.\left(R_{x}\right)\right)$ and radio (transmit $\left.\left(T_{x}\right)\right)$ only. On the other hand, sensors should be capable of acoustic (transmit $\left(T_{x}\right)$ \& receive $\left(R_{x}\right)$ ) and radio (receive $\left(R_{x}\right)$ ) only. Considering most of the practical applications, this assumption is considered pragmatic.

For the acoustic velocity, we are using $v_{A}$ in our mathematical model, which depends on several factors and how these factors affect $v_{A}$ is left for future investigation. However, in our approach, we are able to measure the time in between transmission and reception using radio signals more accurately.

Furthermore, our model has very low overhead. Fig. 3 shows the sequence of action that each node performs; where at the end only one message with the value of $T_{i j(\text { travel })}$ and sensor ID is transferred via acoustic signal from sensor to beacon for distance calculation.

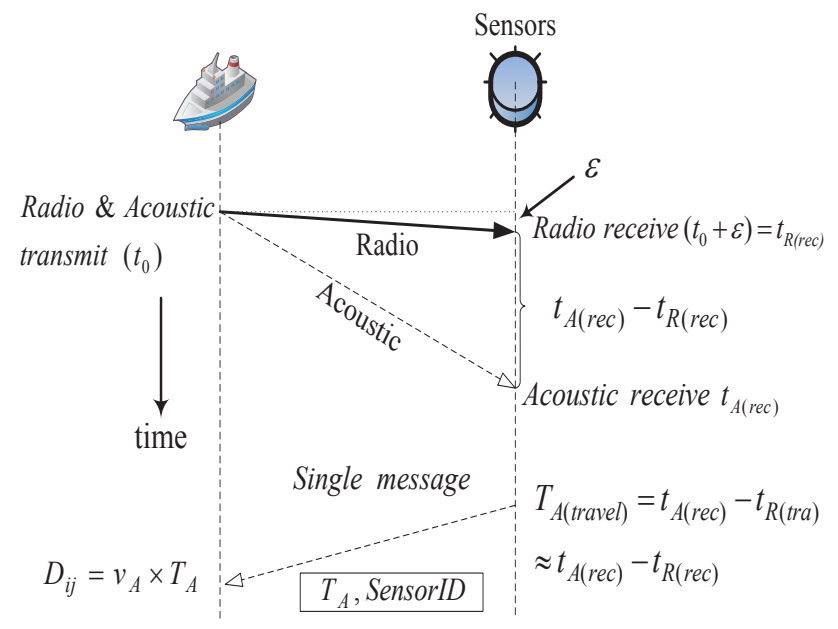

Fig. 3. Message transmission for distance calculation

\section{B. Error Generation}

Our proposed technique incorporates less error in inters node distance measurement because multipath fading has insignificant impact. As stated, sensors are only detecting the arrival of signals instead of decoding a message from its received signal strength. As soon as the shortest Euclidean distance is travelled - signal can be detected by the sensors and used for timing. Hence, the obvious effect of multipath fading in the radio and acoustic signal propagation will be minimal on the timing measurement. With this, a more accurate acoustic speed would give a better distance measurement resulting in coordinates estimate with less error. It is worth mentioning that proposed approach does not use bouncing technique or signal strength to compute distances as they suffer from multipath fading.

\section{SimUlation ReSUlts AND Discussions}

In order to validate the mathematical model, the proposed method has been simulated using Matlab, as it provides procedures to generate numbers in Gaussian distribution. A group of three sensors are placed at $(0,0,0)$, $(0,75,0)$, and $(80,40,0)$ and the mobile beacon is moved randomly in a plane, which is parallel to the bottom plane where the sensors are. The coordinates of the sensors are randomly chosen, while for computational simplicity one of the sensors is placed at the origin and the other one on the axis of the problem domain as discussed in Section IV. While computing the coordinates of the sensors $S_{2}$ and $S_{3}$ with respect to $S_{1}$, Gaussian noise is added with the true Euclidean distances between beacon and sensors.

To get distance measurement from six different positions of the beacon, it has been randomly moved around to six different coordinates within close proximity. However, mobility of the sensors is not considered in the proposed mathematical model. Fig. 4 shows the computed coordinates of the sensors with mean distance.

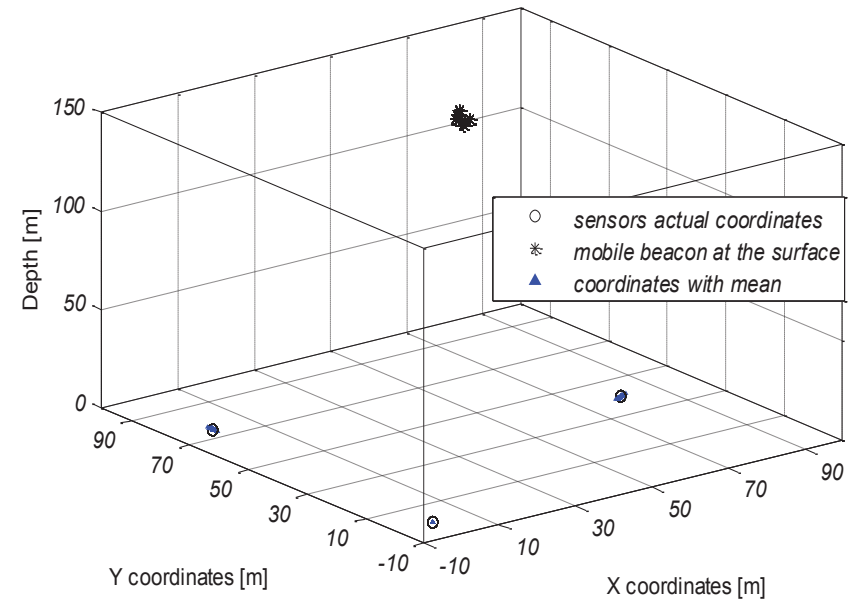

Fig. 4. Calculated sensors positions with proposed method 
Errors in coordinates for sensors $S_{2}$ and $S_{3}$ are shown in Fig. 5 and Fig. 6 respectively for 100 iterations. It should be noted that sensor $S_{1}$ is placed at the reference coordinate $(0,0,0)$; hence producing no error in coordinates determination for $S_{1}$, moreover $S_{2}$ and $S_{3}$ are computed with respect to $S_{1}$.

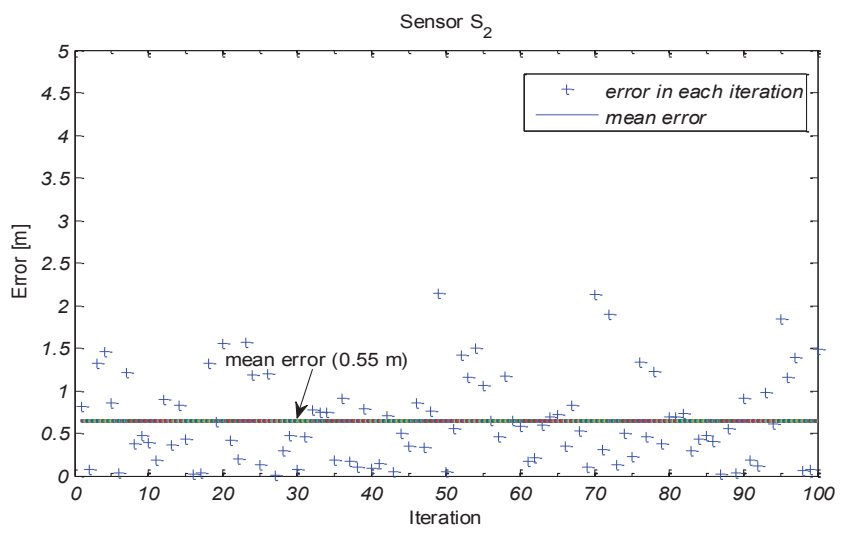

Fig. 5. : Distance errors of sensor $S_{2}$ from the original position

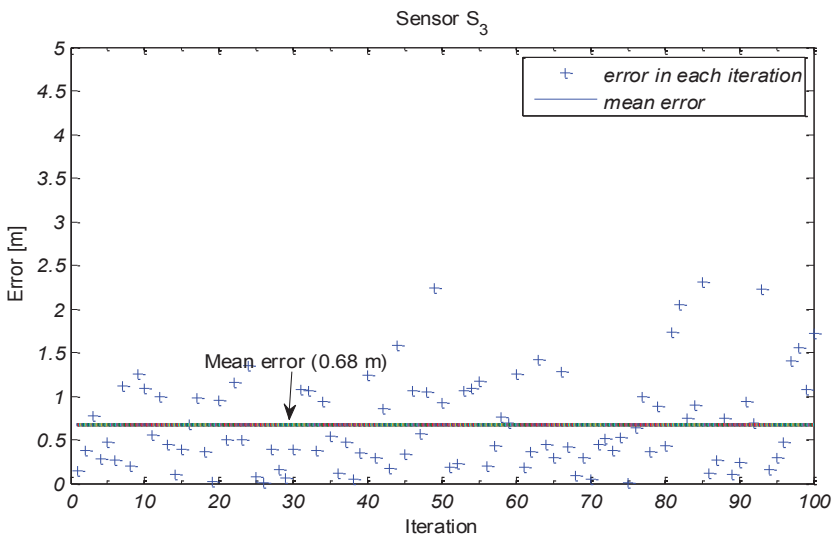

Fig. 6. Distance errors of sensor $S_{3}$ from the original position

TABLE IV compares the positional error of sensor $S_{2}$ and $S_{3}$ when distances between the beacon and sensors are true Euclidean as well as with Gaussian noise. Positional error generates with true Euclidean is almost negligible which validates the proposed mathematical model. Besides, error with Gaussian noise is within acceptable range considering $150 \mathrm{~m}$ water column and usual physical sizes of the sensors. It also shows that the mean error is $0.55 \mathrm{~m}$ and $0.68 \mathrm{~m}$ with standard deviation of 0.469 and 0.559 respectively.

TABLE IV. GENERATION OF ERRORS

\begin{tabular}{|c|c|c|c|}
\hline $\begin{array}{c}\text { Sensors } \\
\text { (unknown } \\
\text { coordinates }\end{array}$ & $\begin{array}{c}\text { Error (m) } \\
\text { (with true } \\
\text { Euclidean } \\
\text { distances) }\end{array}$ & $\begin{array}{c}\text { Mean error (m) } \\
\text { (with Gaussian } \\
\text { noise) }\end{array}$ & $\begin{array}{c}\text { Standard } \\
\text { deviation of } \\
\text { error } \\
\text { distribution }\end{array}$ \\
\hline $\mathrm{S}_{2}$ & $1.06 \times 10^{-10}$ & 0.55 & 0.469 \\
\hline $\mathrm{S}_{3}$ & $2.75 \times 10^{-10}$ & 0.68 & 0.559 \\
\hline
\end{tabular}

\section{CONCLUSIONS}

In this paper we presented a mathematical model to determine the coordinates of submerged sensors using single beacon. The method computes the coordinates with respect to the beacon and sensor node that alleviates a number of problems in the domain of localization. Besides, our distance measurement model and technique contributes minimum error and potentially avoid multipath fading effects in localization. Simulation results validate that proposed mathematical model generates negligible error in coordinate determination of the sensors when distances between beacon and sensors are true Euclidean distance. It also shows that coordinates are within acceptable error range when Gaussian noise is applied to distance determination.

In future work we plan to consider involuntary mobility of the sensors due to currents in the proposed model.

\section{REFERENCES}

[1] H. P. Tan, R. Diamant, W. K. G. Seah, and M. Waldmeyer, "A survey of techniques and challenges in underwater localization," Ocean Engineering, vol. 38, pp. 1663-1676, 2011.

[2] J. H. Cui, J. Kong, M. Gerla, and S. Zhou, "The challenges of building mobile underwater wireless networks for aquatic applications," Network, IEEE, vol. 20, pp. 12-18, 2006.

[3] P. Xie, J. H. Cui, and L. Lao, "VBF: vector-based forwarding protocol for underwater sensor networks," Networking 2006. Networking Technologies, Services, and Protocols; Performance of Computer and Communication Networks; Mobile and Wireless Communications Systems, pp. 1216-1221, 2006.

[4] Z. Zhou, J. H. Cui, and S. Zhou, "Efficient localization for large-scale underwater sensor networks," Ad Hoc Networks, vol. 8, pp. 267-279, 2010

[5] T. C. Austin, R. P. Stokey, and K. M. Sharp, "PARADIGM: a buoybased system for AUV navigation and tracking," in OCEANS 2000 MTS/IEEE Conference and Exhibition, 2000, pp. 935-938 vol.2.

[6] J. C. Prieto, A. R. Jiménez, J. Guevara, J. L. Ealo, F. Seco, J. O. Roa and F. Ramos, "Performance evaluation of 3D-LOCUS advanced acoustic LPS," Instrumentation and Measurement, IEEE Transactions on, vol. 58, pp. 2385-2395, 2009.

[7] P. Duff and H. Muller, "Autocalibration algorithm for ultrasonic location systems," in Wearable Computers, 2003. Proceedings. Seventh IEEE International Symposium on, 2003, pp. 62-68.

[8] E. Olson, J. Leonard, and S. Teller, "Robust range-only beacon localization," in Autonomous Underwater Vehicles, 2004 IEEE/OES, 2004, pp. 66-75.

[9] J. Guevara, A. R. Jimenez, A. S. Morse, J. Fang, J. C. Prieto, and F. Seco, "Auto-localization in Local Positioning Systems: A closedform range-only solution," in Industrial Electronics (ISIE), 2010 IEEE International Symposium on, 2010, pp. 2834-2840.

[10] J. Guevara, A. Jiménez, J. Prieto, and F. Seco, "Auto-localization algorithm for local positioning systems," Ad Hoc Networks, 2012.

[11] X. Che, I. Wells, G. Dickers, P. Kear, and X. Gong, "Re-evaluation of RF electromagnetic communication in underwater sensor networks," Communications Magazine, IEEE, vol. 48, pp. 143-151, 2010.

[12] I. Vasilescu, K. Kotay, D. Rus, M. Dunbabin, and P. Corke, "Data collection, storage, and retrieval with an underwater sensor network," in Proceedings of the 3rd international conference on Embedded networked sensor systems, 2005, pp. 154-165. 